\title{
Nonlinear mechanics of cracks subjected to indentation 1
}

\author{
A.P.S. Selvadurai
}

\begin{abstract}
The paper presents the application of a boundary element technique to study the behaviour of plane cracks that are located at corner regions of an elastic solid and open during indentation. In particular, the surfaces of the planes on which indentation takes place also exhibit Coulomb frictional responses and degradation in the friction angle with plastic energy dissipation. An incremental boundary element formulation, in which special singularity elements model the behaviour at the crack tip, is used to examine the crack problems. The methodology is applied to investigate the mode I stress intensity factor at the crack tip located at the base of a V-notch in a test specimen.
\end{abstract}

Key words: indented cracks, boundary element modelling, Coulomb friction, stress intensity factors.

Résumé : Cet article traite de l'utilisation de la technique des éléments limites pour étudier le comportement de fissures planes situées dans les coins d'un solide élastique et qui s'ouvrent durant le retrait. Plus particulièrement, les surfaces planes où a lieu le retrait démontrent aussi des comportements de frottement solide et une dégradation de l'angle de frottement avec une dissipation plastique de l'énergie. Une formulation incrémentielle d'éléments limites est utilisée pour étudier les problèmes de fissures lorsque des éléments singuliers spéciaux sont utilisés pour modéliser le comportement à l'extrémité des fissures. La méthode est utilisée pour étudier le facteur d'intensité des contraintes de Mode I à l'extrémité de fissures situées à la base d'une encoche en $\mathrm{V}$ dans un spécimen d'essai.

Mots clés: fissures en retrait, modélisation des éléments limite, frottement solide, facteurs d'intensité des contraintes.

[Traduit par la Rédaction]

\section{Introduction}

The stress analysis of flaws, such as cracks and inhomogeneities located in brittle solids (composites, ceramic materials, concrete, rocks, and so on), is important in assessing fracture and failure evolution in these materials. In classical treatments of the behaviour of such defects, the brittle medium is assumed to be elastic and the loads are applied in a manner that does not induce any nonlinear effects, either within the crack region or within the regions in contact (Murakami 1987; Sih 1991; Broberg 1999). The extension of these studies to include nonclassical phenomena, including interactions between the crack faces and closure of the crack tip, becomes important in a number of areas of application, including materials science, civil engineering, and geomechanics, where either alterations in material properties or dominant induced compressive loads normal to the crack surfaces can initiate interaction between the crack surfaces. Such interactions are generally nonlinear, and the presence of the nonlinearity in the mechanics of the contact zone makes the analytical approach to these

Received 19 July 2005. Revision accepted 23 January 2006. Published on the NRC Research Press Web site at http://cjce.nrc.ca/ on 29 August 2006.

A.P.S. Selvadurai. Department of Civil Engineering and Applied Mechanics, McGill University, Montreal, QC H3A 2K6, Canada (e-mail: patrick.selvadurai@mcgill.ca).

Written discussion of this article is welcomed and will be received by the Editor until 31 October 2006.

${ }^{1}$ This article is one of a selection of papers published in this Special Issue honouring Dr. M. Saeed Mirza. crack problems quite restrictive. The analytical study of crack problems becomes almost intractable when complicated forms of frictional contact phenomena, influence of separation and slip zones, interaction of contacting regions with neighbouring cracks, etc., need to be considered. In materials engineering in particular, interfaces between geomaterial regions can exhibit a variety of nonlinear phenomena, including Coulomb friction, finite friction, interface plasticity, interface damage, asperity degradation, viscoplasticity, and creep (Michałowski and Mroz 1978; Selvadurai and Voyiadjis 1986; Selvadurai and Boulon 1995; Darve 1990; Desai 2000). Computational approaches are therefore of particular interest in the study of contact phenomena where interface nonlinearity becomes a dominant feature. Extensive advances have been made in the application of finite element techniques to the study of contact phenomena associated with nonlinear interfaces. Detailed accounts of these developments are given by Zienkiewicz and Taylor (2000), Wriggers and Wagner (2000), and Willner (2003), among others. In this paper, however, the boundary element method is used to examine contact problems dealing with nonlinear effects at the contact zone. The boundary element method is particularly useful when nonlinear contact phenomena are restricted to predefined contact zones. Andersson (1981) and Andersson and Allan-Persson (1983) were some of the earliest to use boundary element techniques to study frictional contact problems. Selvadurai and Au $(1985,1988,1989)$ and Selvadurai (1991, $1993,1995,2005)$ also used these procedures to examine the influence of interface nonlinearity on the behaviour of planar cracks located in elastic media. Applications of the boundary element method to the study of crack extension in brittle elastic media are also given by Aliabadi (1997), Selvadurai and ten Busschen (1995), and Selvadurai (1994, 2000). 
Fig. 1. Opening of a crack at the base of a notch by the frictional indentation with a rigid wedge.

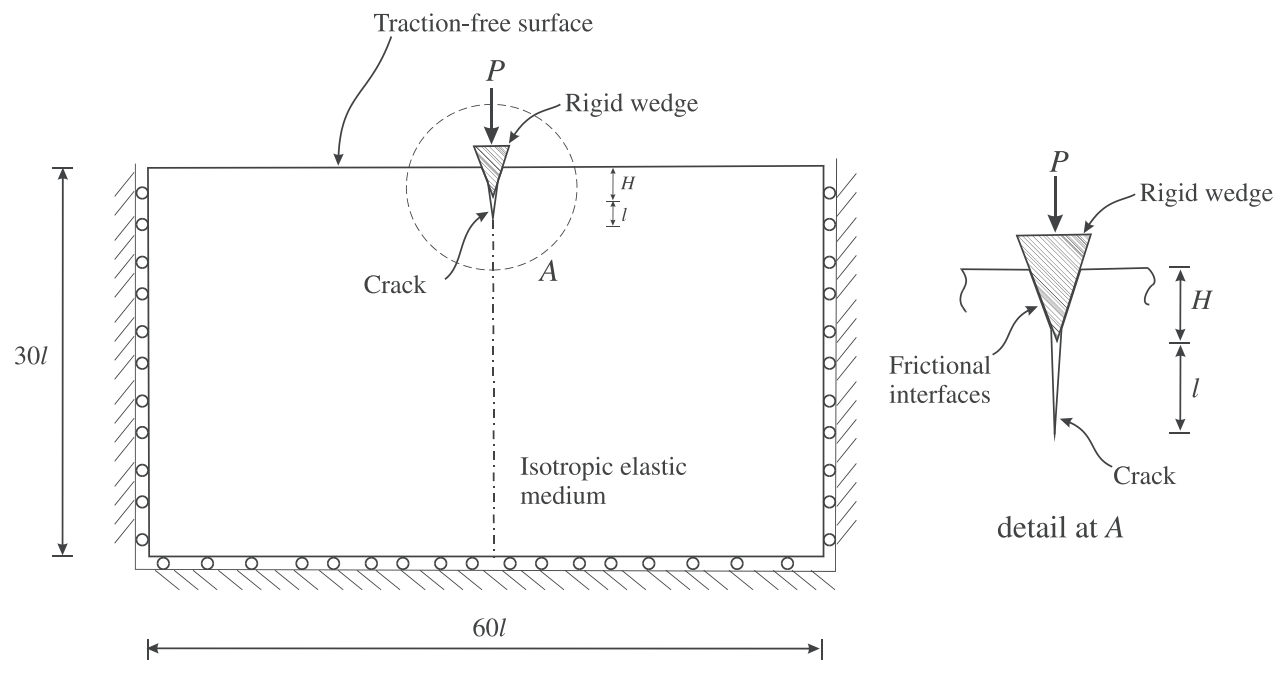

This paper considers an incremental boundary element approach for modelling the mechanics of cracks loaded by wedgetype indenters that exhibit Coulomb friction on the contacting surfaces (Fig. 1). The frictional effects continue to materialize as the wedge indentation forces exert additional stresses in the interface region. For the class of wedge indentation problems considered in this paper, the tips of the cracks remain open throughout the application of the load to the wedge indenter. This provides a plausible model for evaluating the stress intensity factors at the crack tip, without ambiguity.

\section{An incremental boundary element formulation}

The boundary element modelling of elastic continua is a wellestablished computational procedure (Brebbia et al. 1984; Gaul et al. 2003). Because the treatment of nonlinear effects at contacting surfaces has to be approached in an incremental fashion, a brief outline is given here of the incremental form of the boundary integral equation applicable to an elastic medium without body forces. Considering the source point $(P)$, we have

$$
\begin{aligned}
C_{i j}(P) \dot{u}_{j}(P)+\int_{S} T_{i j}^{*}(P, Q) \dot{u}_{j}(Q) \mathrm{d} S & \\
& =\int_{S} U_{i j}^{*}(P, Q) \dot{t}_{j}(Q) \mathrm{d} S
\end{aligned}
$$

where $(Q)$ denotes a general field point; $\dot{u}_{j}(Q)$ and $\dot{t}_{j}(Q)$ are the $j$ th components of the incremental displacements and tractions, respectively; $S$ is the boundary of the body; $C_{i j}(P)$ is a constant (equal to 0 if $(P)$ is outside the body, equal to $\delta_{i j}$ (Kronecker's delta function) if $(P)$ is inside the body, equal to $\delta_{i j} / 2$ if $(P)$ is located at a smooth boundary, and is a function of the discontinuity at a corner and Poisson's ratio if $(P)$ is located at a corner); and $i$ and $j$ are $(1,2)$ or $(x, y)$ for two-dimensional problems. Also in eq. [1], $U_{i j}^{*}(P, Q)$ and $T_{i j}^{*}(P, Q)$ are, respectively, the fundamental solutions applicable to the displacements and tractions and are given by

[2] $U_{i j}^{*}(P, Q)=\frac{1}{8 \pi G(1-v)}\left[(3-4 v) \delta_{i j} \ln \left(\frac{1}{r}\right)+r_{, i} r_{, j}\right]$

and

$$
\begin{aligned}
T_{i j}^{*}(P, Q)= & -\frac{1}{4 \pi(1-v) r}\left\{\left[(1-2 v) \delta_{i j}\right.\right. \\
& \left.\left.+2 r_{, i} r_{, j}\right] r_{, n}-(1-2 v)\left[r_{, i} n_{j}-r_{, j} n_{i}\right]\right\}
\end{aligned}
$$

respectively, where $G$ and $v$ are the shear modulus and Poisson's ratio of the medium, respectively; $r$ is the distance between the source $(P)$ and the field $(Q)$ points; and $n_{i}$ is the $i$ th component of the outward unit normal vector to $S$. The boundary integral equation can be reduced to a matrix equation by discretizing the boundary $S$ into elements with a piecewise continuous variation in the displacements and tractions over the element. Considering all locations of $(P)$ on $S$, one can obtain a system matrix equation relating the displacements and tractions on the boundary. For a well-posed problem, the boundary conditions should be prescribed as follows:

(a) on a boundary $S_{1}$, with known displacements $\bar{u}_{i}$,

$$
\text { [4] } \quad \dot{u}_{i}=\bar{u}_{i}
$$

(b) on a boundary $S_{2}$, with known tractions $\bar{t}_{i}$,

$$
\text { [5] } \dot{t}_{i}=\bar{t}_{i}
$$

(c) on a boundary $S_{3}$, where there is coupling between the displacement and the tractions,

$$
\dot{t}_{i}=E_{i j}^{(\mathrm{ep})}\left(\dot{u}_{j}^{+}-\dot{u}_{j}^{-}\right)
$$

where $E_{i j}^{(\mathrm{ep})}$ is a nonlinear constitutive relationship at the boundary; and the term in parentheses refers to the incremental 
relative displacement at the interface. It is also possible to introduce into eq. [6] an initial traction state derived through consideration of a previous history of loading. The boundary conditions (eqs. [4]-[6]) should make up the complete boundary (i.e., $S=S_{1} \cup S_{2} \cup S_{3}$ ). Applying boundary conditions of eqs. [4] and [5] to the boundary element method system matrix and omitting the boundary condition of eq. [6], we can write

$$
\begin{aligned}
{\left[\begin{array}{ll}
-g_{11} & h_{12} \\
-g_{21} & h_{22} \\
-g_{31} & h_{32}
\end{array}\right]\left\{\begin{array}{c}
\dot{t}_{1} \\
\dot{u}_{2}
\end{array}\right\} } \\
+\left[\begin{array}{ll}
h_{13} & -g_{13} \\
h_{23} & -g_{23} \\
h_{33} & -g_{33}
\end{array}\right]\left\{\begin{array}{c}
\dot{u}_{3} \\
\dot{t}_{3}
\end{array}\right\}=\dot{f}\left\{\begin{array}{l}
B_{1} \\
B_{2} \\
B_{3}
\end{array}\right\}
\end{aligned}
$$

where $\dot{f}$ is a loading factor; $\{\boldsymbol{B}\}$ is the vector from prescribed boundary values; $\left[h_{i j}\right]$ and $\left[g_{i j}\right]$ are the coefficients matrices from the fundamental solutions; and the suffixes indicate the location of the boundaries $S_{1}, S_{2}$, and $S_{3}$. Equation [7] cannot be solved, because the boundary condition on $S_{3}$ has not been applied. This is, however, the boundary element method system equation, with the boundary condition on $S_{3}$ to be determined.

\section{Interface responses}

The study of interface responses has been a topic of interest to scientists and engineers alike and can be attempted at a variety of levels, ranging from local-scale models to phenomenological approaches. The former consider a level of refinement that is not accounted for in a purely phenomenological approach (Belak 1993; Bushan 1995). This level of refinement is a drawback, because it requires the determination of sophisticated constitutive parameters through experimentation; furthermore, the interpretation of such experiments can be attempted only through a set of phenomenological models themselves. For engineering purposes, therefore, it is desirable to adopt a purely phenomenological approach to formulate an interface constitutive response. The elementary models of interface behaviour that assume either completely smooth or bonded conditions represent extremes of the phenomenological approach. Other nonlinear forms of interface responses are derived from consideration of conventional models of Coulomb friction, plasticity, and dilatant phenomena that account for a local-scale structure and the incorporation of damage and degradation to account for deterioration of the interface with progressive wear (Johnson 1985; Selvadurai and Yu 2005).

This paper considers a treatment of the interface response that can be represented by the dilation models proposed by Plesha (1987), Selvadurai and Au (1988), and Nguyen and Selvadurai (1998). For example, by assuming that the interface is aligned with the $x$ axis, we can show a relative displacement occurring between both sides of the interface. Given the general framework originally proposed by Fredriksson (1976), the incremental form of the relative displacement can be expressed as

[8] $\quad \dot{R}_{i}=\dot{u}_{i}\left(x, 0^{+}\right)-\dot{u}_{i}\left(x, 0^{-}\right)$

which can be considered a combination of elastic and plastic components. For example,

[9] $\quad \dot{R}_{i}=\dot{R}_{i}^{(\mathrm{e})}+\dot{R}_{i}^{(\mathrm{p})}$
Fig. 2. The dilatant interface.

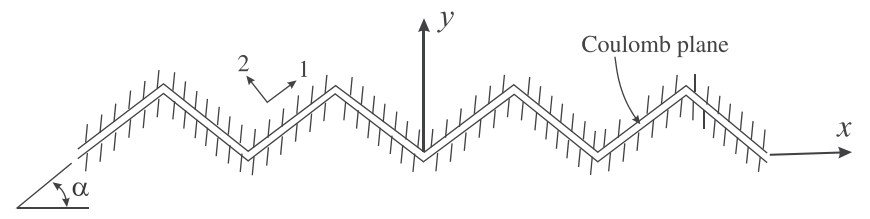

where (e) and (p) refer to the elastic and plastic components, respectively. The elastic displacements at the interface can be determined from the following incremental elastic constitutive relationship:

$$
\dot{t}_{i}=E_{i j}^{(\mathrm{e})} \dot{R}_{j}^{(\mathrm{e})}
$$

where $E_{i j}^{(\mathrm{e})}$ is the elastic stiffnesses at the interface. Under the application of loads, the constitutive relation defined by eq. [10] is valid only if the traction field at the interface does not violate the yield criterion applicable to the interface. Considering the dilative nature of the interface, we adopt a failure criterion of the form

$$
\begin{aligned}
F\left(t_{i}\right)=\left\{\left(t_{x} \cos \alpha+t_{y} \sin \alpha\right)^{2}\right. & \}^{1 / 2} \\
& +\mu\left(t_{x} \sin \alpha-t_{y} \cos \alpha\right)
\end{aligned}
$$

where $\mu$ is the coefficient of friction at the interface of the contacting planes; and $\alpha$ is the asperity angle, as defined by Fig. 2. When the asperity angle reduces to zero, eq. [11] reduces to Coulomb frictional behaviour, i.e.,

$$
F\left(t_{i}\right)=\left|t_{x}\right|-\mu t_{y}
$$

At yield, the irreversible plastic deformation can be obtained from a flow rule similar to that used in conventional plasticity theory (Drucker 1966; Chen 1975; Davis and Selvadurai 2002)

$$
\dot{R}_{i}^{(\mathrm{p})}=\left\{\begin{array}{l}
0 \quad \text { if } \quad F<0 \quad \text { or } \quad \dot{F}<0 \\
\dot{\lambda} \frac{\partial \Phi}{\partial t_{i}} \quad \text { if } \quad F=\dot{F}=0
\end{array}\right.
$$

where $\dot{\lambda}$ is a plastic multiplier; and $\Phi$ is the plastic potential function, which is given by

$$
\Phi=\left\{\left(t_{x} \cos \alpha+t_{y} \sin \alpha\right)^{2}\right\}^{1 / 2}
$$

If we adopt conventional formulations of the theory of plasticity, we can write the incremental form of the elastoplastic stressstrain relationship as

$$
\text { [15] } \quad \dot{t}_{i}=E_{i j}^{(\mathrm{ep})} \dot{R}_{j}
$$

where $E_{i j}^{(\mathrm{ep})}$ is the elastoplastic stiffness at the interface, which is given by

$$
E_{i j}^{(\mathrm{ep})}=E_{i j}^{(\mathrm{e})}-\frac{1}{\psi} \frac{\partial F}{\partial t_{l}} E_{l j}^{(\mathrm{e})} E_{i m}^{(\mathrm{e})} \frac{\partial \Phi}{\partial t_{m}}
$$

and $\psi$ is defined as

$$
\psi=\frac{\partial F}{\partial t_{l}} E_{l m}^{(\mathrm{e})} \frac{\partial \Phi}{\partial t_{m}}-\frac{\partial F}{\partial W_{x}^{(\mathrm{p})}} \dot{W}_{x}^{(\mathrm{p})}
$$


where $W_{x}^{(\mathrm{p})}$ is given by $t_{x} \dot{R}_{x}^{(\mathrm{p})}$, which is the term corresponding to the plastic work of the tractions tangential to the failure plane. This plastic work is responsible for the degradation of the asperity angle, which, for example, can be expressed in the form

$$
\text { [18] } \quad \alpha=\alpha_{0} \exp \left\{-c W_{x}^{(\mathrm{p})}\right\}
$$

where $\alpha_{0}$ is an initial asperity teeth angle, and $c$ is referred to as a degradation constant. In instances where the elastic stiffnesses at the interface have only the normal and shear components (i.e., $E_{i j}^{(\mathrm{e})}=0$ when $i \neq j$ ),

$$
\begin{aligned}
& E_{11}^{(\mathrm{ep})}=E_{11}^{(\mathrm{e})}\left\{1-\frac{1}{\psi} E_{11}^{(\mathrm{e})} \frac{\partial F}{\partial t_{x}} \frac{\partial \Phi}{\partial t_{x}}\right\} \\
& E_{22}^{(\mathrm{ep})}=E_{22}^{(\mathrm{e})}\left\{1-\frac{1}{\psi} E_{22}^{(\mathrm{e})} \frac{\partial F}{\partial t_{y}} \frac{\partial \Phi}{\partial t_{y}}\right\}
\end{aligned}
$$

and

$$
\begin{gathered}
\frac{E_{12}^{(\mathrm{ep})}}{\left(\partial F / \partial t_{y}\right) /\left(\partial \Phi / \partial t_{x}\right)}=\frac{E_{21}^{(\mathrm{ep})}}{\left(\partial F / \partial t_{x}\right) /\left(\partial \Phi / \partial t_{y}\right)} \\
=\frac{1}{\psi} E_{11}^{(\mathrm{e})} E_{22}^{(\mathrm{e})}
\end{gathered}
$$

which is applicable to a dilatant interface model. For a Coulomb frictional interface, the limiting case of eqs. [19]-[21] gives

$$
E_{11}^{(\mathrm{ep})}=E_{22}^{(\mathrm{ep})}=0
$$

and

$$
\frac{E_{12}^{(\mathrm{ep})}}{\mu \sin \left(-t_{x}\right)}=E_{22}^{(\mathrm{ep})}
$$

Using the above relationships, one can arrive at the exact Coulomb relationship (Michałowski and Mroz 1978; Desai and Siriwardane 1984; Lubliner 1990; Davis and Selvadurai 2002):

$$
\dot{t}_{x}=-\mu \sin \left(t_{x}\right) \dot{t}_{y}
$$

Equations [19]-[24] are applicable for the case where the interface is aligned with the $x$ axis. These developments can be extended to the situation in which the interface can be aligned at an arbitrary orientation to the reference coordinate system.

\subsection{Contact and separation processes}

During incremental analysis of an interface contact problem, within an increment of loading, processes such as separation, reestablishment of contact, slip, and adhesion can occur in distinct regions of the interface. Analytical constraints can be placed on these processes, and we will discuss these, recognizing plausible physical characteristics associated with such responses.

\subsubsection{Separation}

In a loading sequence, the normal tractions at an interface region can become tensile. Because the contact response of admissible interfaces is generally unilateral (i.e., unable to withstand tension) for a region undergoing separation, the total tractions resulting from the summation of the incremental boundary conditions of eq. [5] should be zero.

\subsubsection{Recontact}

A region of a contact zone that has experienced separation can also reestablish contact when the relative normal displacement across the separated interface region is greater than or equal to the initial gap. In such a case, the boundary conditions change from a type given by eq. [5] to the one given by eq. [4].

\subsubsection{Slip}

When the tractions satisfy the failure condition given by eq. [11], slip will occur and the interface condition given by eq. [6] can be applied, with the stiffness coefficients now defined by eq. [16].

\subsubsection{Adhesion}

When the conditions do not violate the failure criterion given by eq. [11], the boundary conditions at the interface can be interpreted through eq. [6], with the stiffness coefficients interpreted appropriately. In a solution scheme, with every increment, all of the above four conditions must be checked to obtain a stable condition at the interface.

\section{Boundary element modelling of crack tip behaviour}

For the discretization of a boundary region that does not contain a crack tip, quadratic elements can be employed. In this case, the variation in the displacements and tractions within the element can be described by

$$
\left.\begin{array}{c}
u_{i} \\
t_{i}
\end{array}\right\}=a_{0}+a_{1} \zeta+a_{2} \zeta^{2}
$$

where $\zeta$ is the local coordinate associated with the element; and $a_{i}(i=0,1,2)$ represents the constant of interpolation. In the definition of the stress state at the crack tip in an elastic medium, however, the stress state is singular, and the corresponding displacements at the crack tip are given by

$$
\begin{aligned}
& \frac{4 G u_{x}}{\sqrt{r / 2 \pi}}=K_{\mathrm{I}}\left[(2 k-1) \cos \frac{\theta}{2}-\cos \frac{3 \theta}{2}\right] \\
& +K_{\mathrm{II}}\left[(2 k+3) \sin \frac{\theta}{2}+\sin \frac{3 \theta}{2}\right] \\
& \frac{4 G u_{y}}{\sqrt{r / 2 \pi}}=K_{\mathrm{I}}\left[(2 k+1) \sin \frac{\theta}{2}-\sin \frac{3 \theta}{2}\right] \\
& +K_{\mathrm{II}}\left[(2 k-3) \cos \frac{\theta}{2}+\cos \frac{3 \theta}{2}\right]
\end{aligned}
$$

where the crack is considered to be aligned parallel to the $x$ axis; $r, \theta$ are the polar coordinates relative to the crack tip; $K_{\mathrm{I}}$ and $K_{\mathrm{II}}$ are the mode I and mode II stress intensity factors for the crackopening and crack-shearing modes, respectively; $k=(3-4 v)$ for plane-strain problems; and $k=(3-v) /(1+v)$, for planestress problems. To capture the order of the stress singularity at the crack tip, Cruse and Wilson (1977) introduced a singular traction boundary element where the traction variations take the form

$$
t_{i}=\frac{c_{0}}{\sqrt{r}}+c_{1}+c_{2} \sqrt{r}
$$


Fig. 3. Node arrangement for calculation of stress intensity factors at a crack tip.

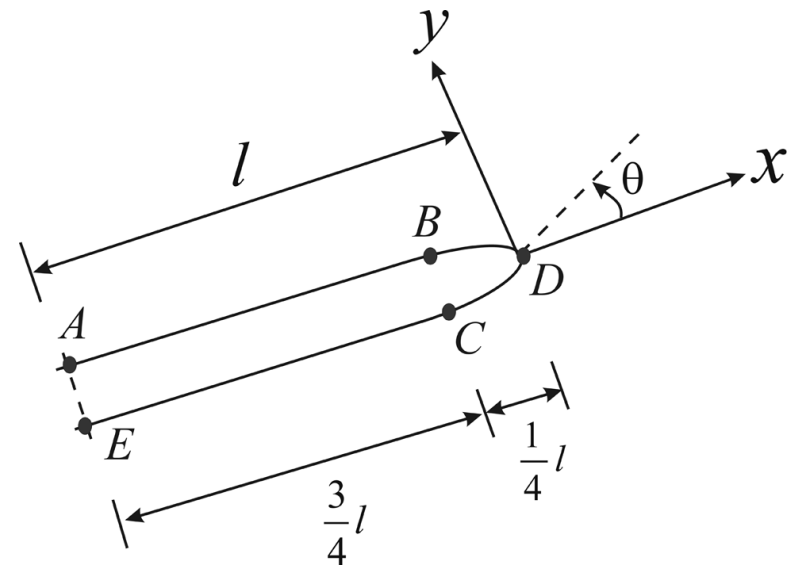

where $c_{i}(i=0,1,2)$ are constants. The accuracy of resulting formulations has been examined in detail, and references to comparison exercises are given by Aliabadi (1997). Other examples are also given by Selvadurai and $\mathrm{Au}(1988,1989)$, Selvadurai and ten Busschen (1995), and Selvadurai (2000).

The incorporation of a special singularity element permits the evaluation of the stress intensity factors at the crack tip. Generally, for the problems with in-plane deformations discussed here, only the mode I and mode II stress intensity factors are relevant. The increments in these stress intensity factors can be determined by a displacement correlation method, which makes use of the increments of the nodal displacements at four locations, A, B, C, and E, and the crack tip D (Fig. 3). The incremental estimates for the stress intensity factors are given by

$$
\begin{aligned}
\dot{K}_{\mathrm{I}}=\frac{2 G}{(k+1)} \sqrt{\frac{2 \pi}{l}}\left\{4\left[\dot{u}_{y}(B)-\dot{u}_{y}(C)\right]\right. & \\
& \left.+\dot{u}_{y}(E)-\dot{u}_{y}(A)\right\}
\end{aligned}
$$

$$
\begin{aligned}
\dot{K}_{\mathrm{II}}=\frac{2 G}{(k+1)} \sqrt{\frac{2 \pi}{l}}\left\{4 \left[\dot{u}_{x}(B)-\right.\right. & \left.\dot{u}_{x}(C)\right] \\
& \left.+\dot{u}_{x}(E)-\dot{u}_{x}(A)\right\}
\end{aligned}
$$

where $l$ is the length of the crack-tip element; and points A, B, $\mathrm{C}, \mathrm{E}$, and D are as indicated in Fig. 3.

\section{Localized iterative solution procedures}

In the solution of the nonlinear interface problem, the conditions on the surface $S_{3}$ need to be determined during the incremental iterative analysis. These conditions can only be determined after the boundary element matrix eq. [7] has been solved. The repeated solution of the boundary element matrix equation can be computationally inefficient, particularly when the general class of three-dimensional problems is examined. A way to improve the efficiency of the solution procedure is to eliminate beforehand the unknowns corresponding to the boundary location on $S_{1}$ and on $S_{2}$. Performing this operation, we obtain

$$
\begin{aligned}
& {\left[\begin{array}{cc}
\nabla_{11} & \bar{h}_{12} \\
0 & \nabla_{22} \\
0 & 0
\end{array}\right]\left\{\begin{array}{l}
\dot{t}_{1} \\
\dot{u}_{2}
\end{array}\right\}} \\
& +\left[\begin{array}{ll}
\bar{h}_{13} & -\bar{g}_{13} \\
\bar{h}_{23} & -\bar{g}_{23} \\
\bar{h}_{33} & -\bar{g}_{33}
\end{array}\right]\left\{\begin{array}{c}
\dot{u}_{3} \\
\dot{t}_{3}
\end{array}\right\}=\dot{f}\left\{\begin{array}{l}
\bar{B}_{1} \\
\bar{B}_{2} \\
\bar{B}_{3}
\end{array}\right\}
\end{aligned}
$$

where $\nabla_{i i}(i=1,2)$ are the upper triangle matrices; and the other matrices $\left[\bar{h}_{i j}\right]$ and $\left[\bar{g}_{i j}\right]$ are the reduced versions of $\left[h_{i j}\right]$ and $\left[g_{i j}\right]$, respectively. Equation [31] can be split into two parts, as follows:

$$
\left[\begin{array}{cc}
\nabla_{11} & \bar{h}_{12} \\
0 & \nabla_{22}
\end{array}\right]\left\{\begin{array}{l}
\dot{t}_{1} \\
\dot{u}_{2}
\end{array}\right\}=\dot{f}\left\{\begin{array}{c}
\bar{B}_{1} \\
\bar{B}_{2} \\
\bar{B}_{3}
\end{array}\right\}-\left[\begin{array}{ll}
\bar{h}_{13} & -\bar{g}_{13} \\
\bar{h}_{23} & -\bar{g}_{23}
\end{array}\right]\left\{\begin{array}{c}
\dot{u}_{3} \\
\dot{t}_{3}
\end{array}\right\}
$$

This is a back-substitution equation for the unknowns $\left\{\dot{T}_{1}\right\}$ and $\left\{\dot{u}_{2}\right\}$. The back substitution can be applied either currently or when the values of $\left\{\dot{u}_{3}\right\}$ and $\left\{\dot{t}_{3}\right\}$ are determined. The computational efficiency of the back-substitution procedure is governed by the size of the domains $S_{1}$ and $S_{2}$. Equation [32] can, however, be used at every iteration and increment step. The third row of eq. [31] can be written as

$$
\left[\bar{h}_{33}\right]\left\{\dot{u}_{3}\right\}-\left[\bar{g}_{33}\right]\left\{\dot{t}_{3}\right\}=\dot{f}\left[\bar{B}_{3}\right]
$$

Equation [33] can be solved together with the interface condition, which can be expressed by

$$
\left[K^{(\mathrm{ep})}\right]\left\{\dot{t}_{3}\right\}=\{0\}
$$

Equation [33] can be regarded as a family of parallel planes in the $\left\{\dot{u}_{3}\right\}$ versus $\left\{\dot{t}_{3}\right\}$ space. The location of the parallel plane is controlled by the increment $\dot{f}$. For an elastic interface, eq. [34] also represents a plane and the intersection of the solutions, as shown in Fig. 4. The solution procedure during an increment follows the sequence $0 \rightarrow 1 \rightarrow 1^{\prime} \rightarrow 2 \rightarrow 2^{\prime} \rightarrow$, etc. The convergence is achieved by assigning an acceptable tolerance to the iteration. In this procedure, the iteration for the condition on $S_{3}$ is performed locally, and the boundary element system matrix is eliminated only once for any number of increments.

\section{A frictional indentation problem}

In this paper, we combine the procedures for describing the nonlinear incremental boundary element formulation, the technique for modelling the crack-tip behaviour, and the localized iterative procedures described in the previous sections to examine certain problems related to the opening of cracks extending from notches located at the boundaries of the elastic regions. The opening of the cracks is due to indentation by a rigid indentor that is subjected to a total force $P$. The parameters required for the computational modelling are the coefficient of friction at the interface region $(\mu)$; the asperity degradation parameter $(c)$; the geometry of the crack in relation to certain characteristic dimensions associated with the corner region $(l / H)$; and the relative magnitudes of the nondimensional shear and normal 
Fig. 4. The iteration procedure for the nonlinear analysis: $(a)$ incremental analysis; $(b)$ iteration within an increment, detail at A.
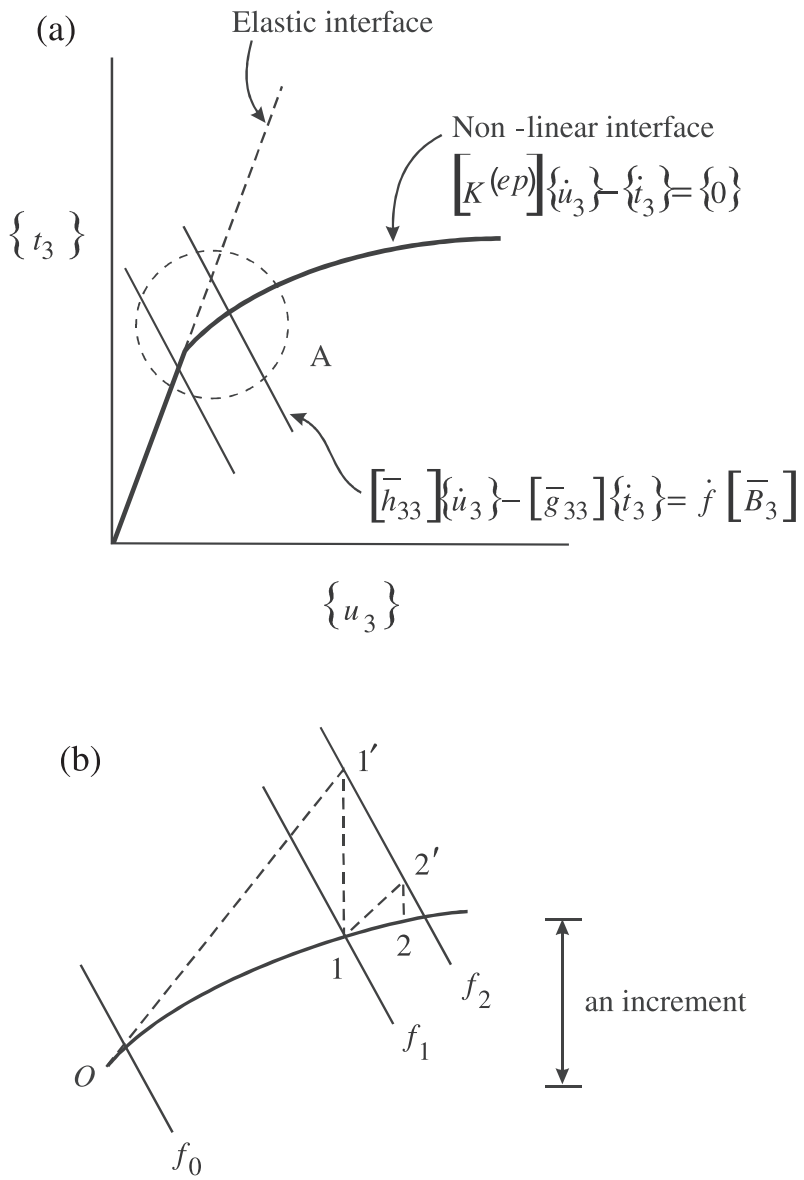

elastic stiffnesses, as defined by $\tilde{E}_{11}^{(\mathrm{e})} / G$ and $\tilde{E}_{22}^{(\mathrm{e})} / G$, where $G$ is the shear modulus of the elastic material. (Note that as per eq. [10], the stiffnesses $E_{11}^{(\mathrm{e})}$ and $E_{22}^{(\mathrm{e})}$ are expressed in units of force/(length) ${ }^{3}$ to provide tractions with units of stress. In the normalization of these stiffnesses, we can define nondimensional parameters $\tilde{E}_{11}^{(\mathrm{e})} / G$ and $\tilde{E}_{22}^{(\mathrm{e})} / G$, where $\tilde{E}_{11}^{(\mathrm{e})}=E_{22}^{(\mathrm{e})} \times 1$ and $\tilde{E}_{22}^{(\mathrm{e})}=E_{22}^{(\mathrm{e})} \times 1$, where unity represents the thickness over which the plane problem is being analysed.)

For purposes of illustration, the following specific values of the interface stiffness parameters and the initial stress state are used in the computations:

$$
\frac{\tilde{E}_{11}^{(\mathrm{e})}}{G}=10^{3} ; \quad \frac{\tilde{E}_{22}^{(\mathrm{e})}}{G}=0.5 \times 10^{3}
$$

The boundary element discretization of the domain is shown in Fig. 5. As a result of the symmetry associated with the indentation of the notch containing the crack by the rigid wedge only the mode I stress intensity factor is developed at the crack tip. Figure 6 illustrates the variation of the normalized mode I stress intensity factor, $K_{\mathrm{I}} / G \sqrt{l}$, with the normalized indentation force, $\left(4 P / G H^{2}\right) \times 10^{3}$, and as a function of the interface friction $\mu$ and the normalized interface asperity degradation parameter $(c / G)=10^{-5}$. Similarly, the results presented in
Fig. 5. Boundary element discretization of the domain.

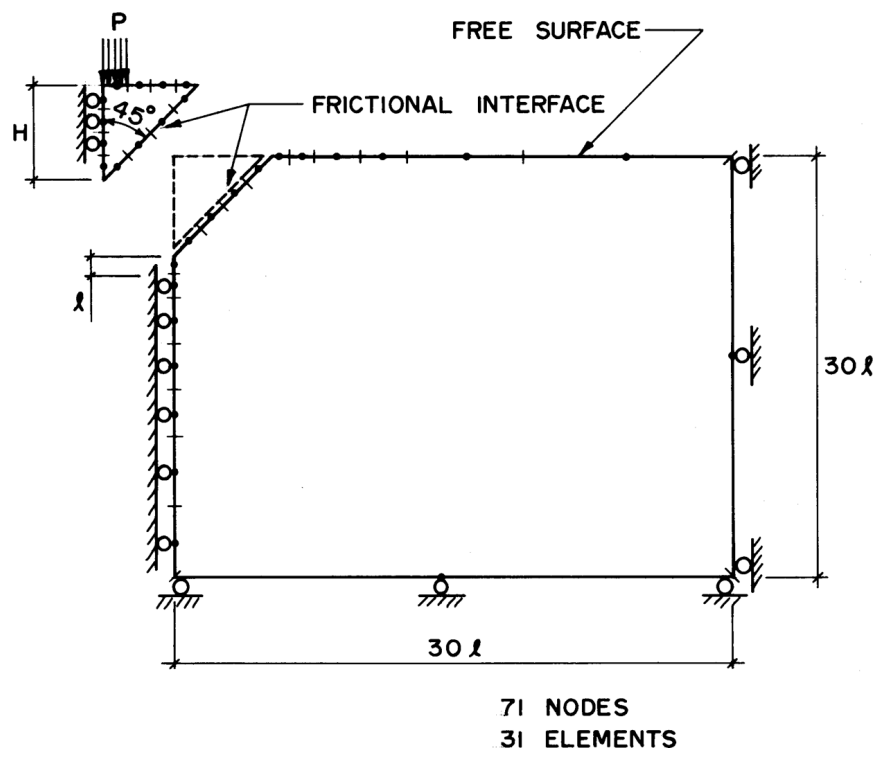

Fig. 7 are applicable to the case in which the normalized interface degradation parameter is increased by an order of magnitude to $\left(c / G=10^{-4}\right)$. As is evident, the interface friction has a significant influence on the magnitude of the normalized stress intensity factor $K_{\mathrm{I}} / G \sqrt{l}$. The limiting bound for the mode I stress intensity factor corresponds to the case of frictionless indentation. The interface degradation parameter also has an influence on the magnitude of the stress intensity factor at the crack tip. As the interface degradation property decreases, the stress intensity factor is amplified for the same value of the friction coefficient. Alternative representations of the influence of the asperity degradation parameter are shown in Figs. 8 and 9 for various values of the relative crack lengths and the friction coefficient. General conclusions cannot be inferred from this data because of differences in the parameters associated with the data. It can be concluded that as the relative crack length increases, the mode I stress intensity factor is reduced. A further observation is that the case involving a completely bonded interface between the faces of the wedge and the indentor does not always represent the condition that contributes to the lowest stress intensity factor. Interfaces that experience degradation can result in the lowering of the stress intensity factor at the crack tip. Finally, we consider an example of the use of the boundary element procedure in examining the mechanics of test procedures that can be used to assess the fracture toughness of brittle elastic materials, the plane problem of the indentation of a surface-notched cylinder containing a basal crack extending in the radial direction and subjected to indentation by a rigid cylinder (Fig. 10). Admittedly, as the contact between the rigid cylinder and the faces of the wedge occurs at a point location, the influences of friction at the contact locations are disregarded. Figure 10 illustrates the manner in which the mode I stress intensity factor at the basal crack is influenced by the relative geometric dimensions of the crack. As with the plane problem in elasticity theory, the stress state is independent of the elasticity characteristics of the medium; consequently, the mode I stress intensity factor is independent of both the elastic modulus and Poisson's ratio of the cylinder. 
Fig. 6. Mode I stress intensity factor at the crack tip extending from a notch.

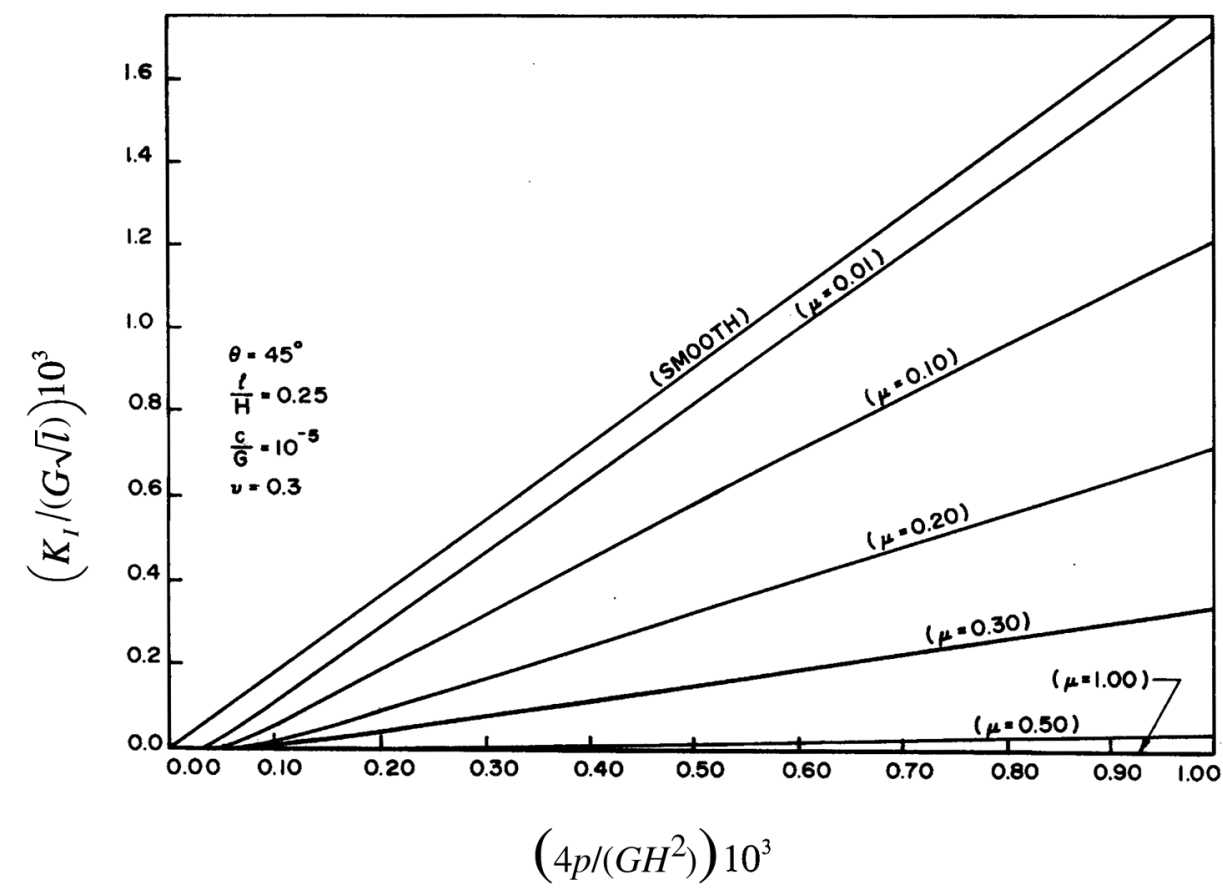

Fig. 7. Mode I stress intensity factor at the crack tip extending from a notch.

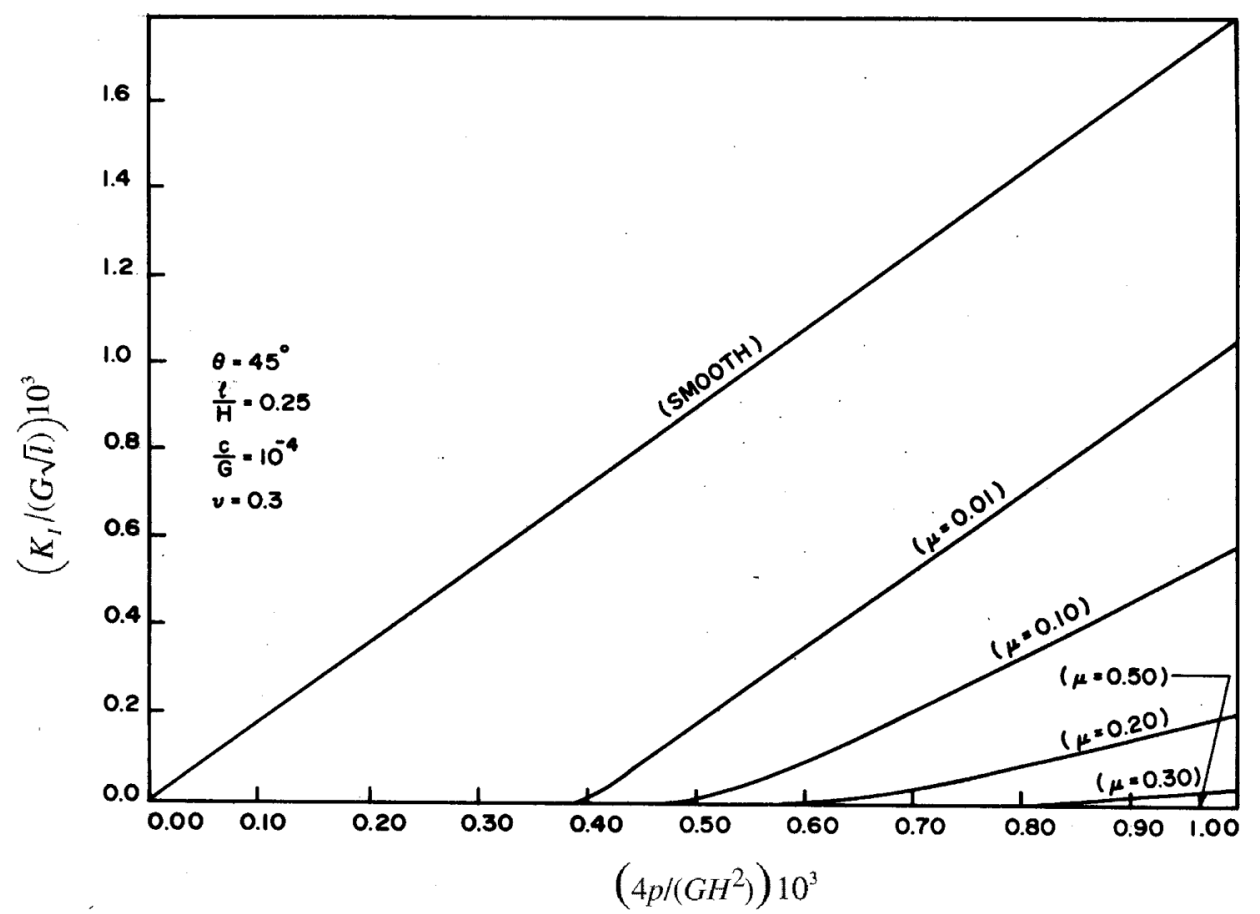

\section{Concluding remarks}

The mechanics of cracks can be influenced by the nonlinear processes that can either be present within the crack region or be initiated as a result of interaction with a contact region in the vicinity of the crack. The problem examined in this paper relates to the latter category, where a crack extending from the root of a notch is subjected to opening action by a rigid wedge in frictional contact with the surfaces of the notch. Because the nonlinear effects are restricted a priori to known surfaces, the analysis of the resulting nonlinear problem can be achieved by taking an incremental boundary element approach. It is found that the result of practical importance to engineering applica- 
Fig. 8. Mode I stress intensity factor at the crack tip extending from a notch.

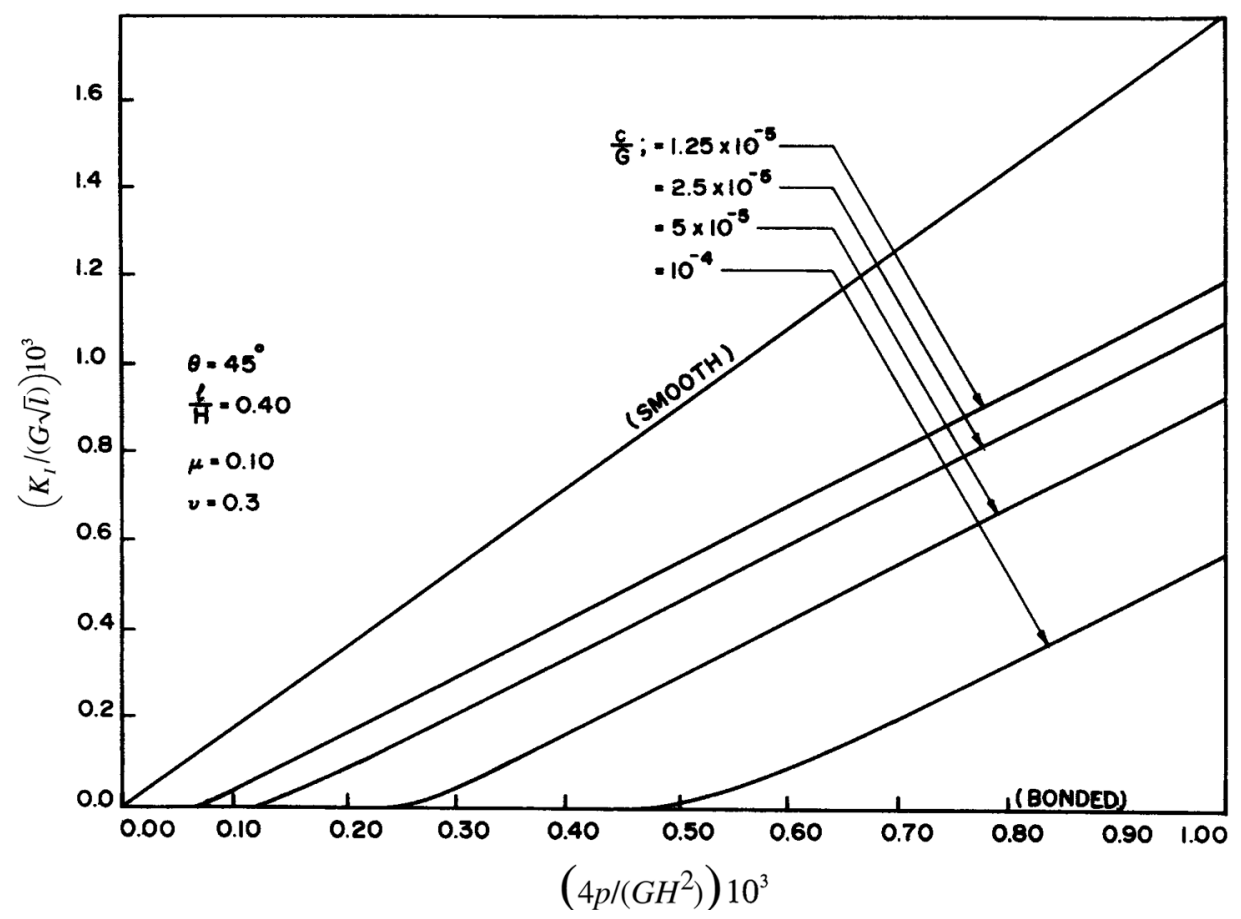

Fig. 9. Mode I stress intensity factor at the crack tip extending from a notch.

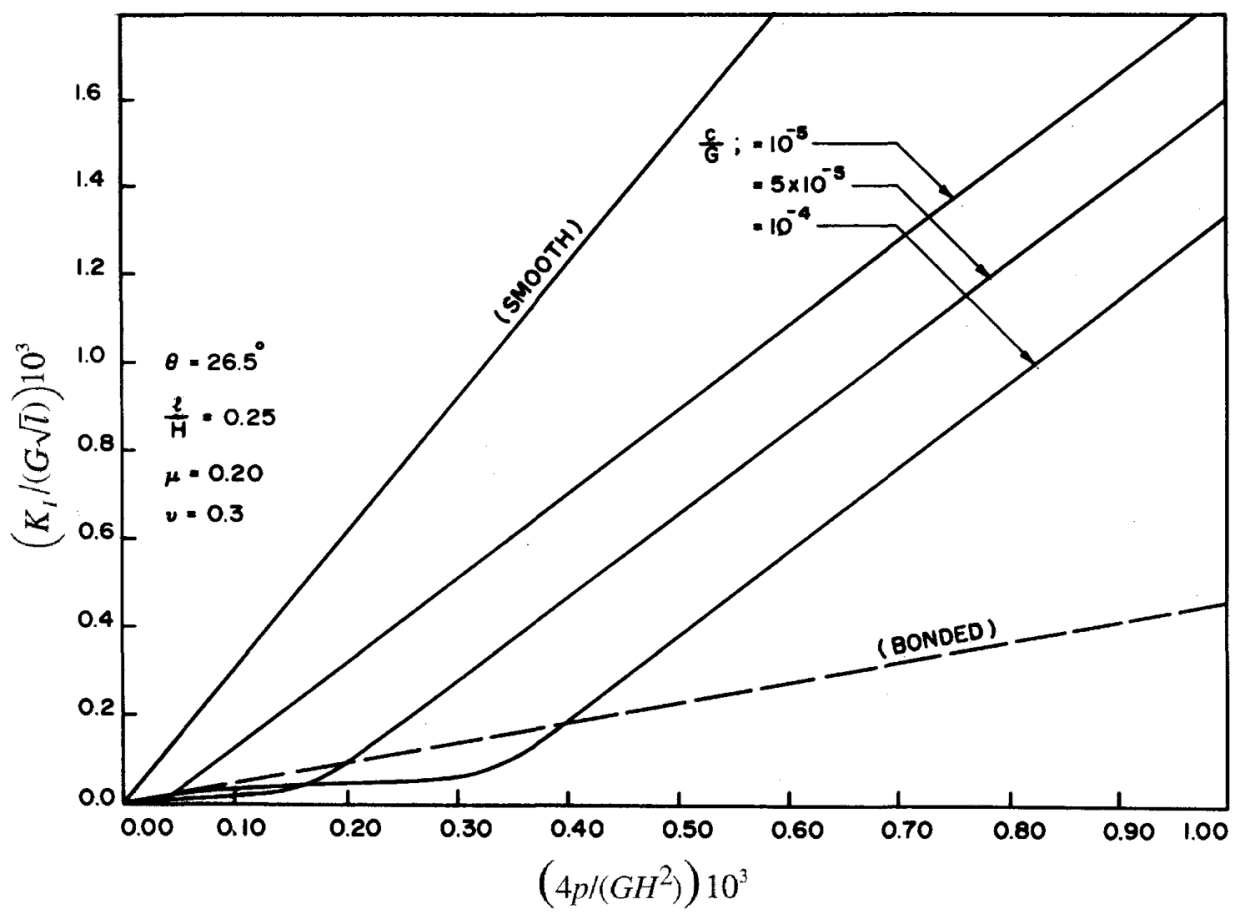

tions, namely, the mode I stress intensity factor at the crack tip, is influenced by the frictional properties of the contact zone and the parameter that describes the deterioration of the friction angle with plastic energy dissipation in shear. In general, the effect of friction on the contact zones is to reduce the stress intensity factor due to indentation that is observed at the basal crack tip. The absence of friction invariably results in a greater separation of the faces of the wedge, resulting in the generation of the greatest magnitude of stress intensity factor, which, for the purely elastic problem, is directly proportional to the applied load. The decay of the frictional characteristics at the contact region also contributes to the increase in the stress in- 
Fig. 10. The indentation of a notched cylinder containing a basal crack.

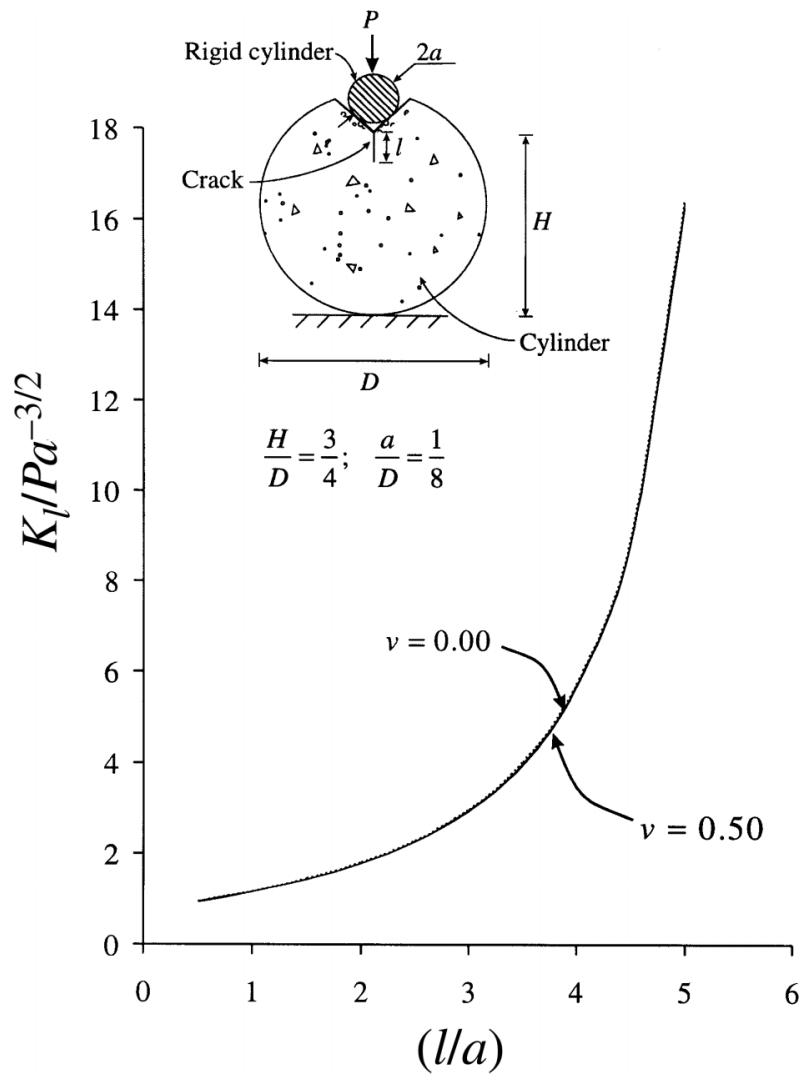

tensity factor at the crack extending from the base of the wedge region.

\section{Acknowledgements}

The paper was prepared during the author's visit to the Institut A f ür Mechanik, Universität Stuttgart, Germany, as the 2003 Max Planck Forschungspreisträger in the Engineering Sciences. The hospitality of the institut is gratefully appreciated. The author is also grateful to Dr. M.C. Au, former research associate, for performing parts of the computational modelling.

\section{References}

Aliabadi, M.H. 1997. Boundary element formulations in fracture mechanics. Applied Mechanics Reviews, 50: 83-96.

Andersson, T. 1981. The boundary element method applied to twodimensional contact problems with friction. In Boundary Element Methods: Proceedings of the 3rd International Seminar, Irvine, California, July. Edited by C.A. Brebbia. Springer-Verlag, Berlin.

Andersson, T., and Allan-Persson, B.G. 1983. The boundary element method applied to two-dimensional contact problems. Ch. 5. In Progress in boundary element methods. Vol 2. Edited by C.A. Brebbia. CML Publications, UK. pp. 136-157.

Belak, J.F. 1993. Nanotribology. Materials Research Society Bulletin, 18: $15-16$.
Brebbia, C.A., Telles, J.C.P., and Wrobel, L.C. 1984. Boundary element techniques: theory and applications in engineering. SpringerVerlag, Berlin.

Broberg, B.K. 1999. Cracks and fracture. Academic Press, San Diego, Calif.

Bushan, D. 1995. Handbook of micro/nanotribology. Vol. 1. CRC Press, Boca Raton, Fla.

Chen, W.-F. 1975. Limit analysis and soil plasticity. Developments in Geotechnical Engineering. Elsevier Science Pub. Co., Amsterdam, Netherlands.

Cruse, T.A., and Wilson, R.B. 1977. Boundary integral equation methods for elastic fracture mechanics. AFOSR-TR-0355.

Darve, F. (Editor). 1990. Geomaterials: constitutive equations and modelling. Routledge, New York.

Davis, R.O., and Selvadurai, A.P.S. 2002. Plasticity and geomechanics. Cambridge University Press, Cambridge, UK.

Desai, C.S. 2000. Mechanics of materials and interfaces: the disturbed state concept. CRC Press, Boca Raton, Fla.

Desai, C.S., and Siriwardane, H.J. 1984. Constitutive laws for engineering materials with emphasis on geologic materials. PrenticeHall, Upper Saddle River, N.J.

Drucker, D.C. 1966. Introduction to the mechanics of deformable solids. McGraw-Hill, New York.

Fredriksson, B. 1976. Finite element solution of surface non-linearities in structural mechanics with special emphasis to contact and fracture mechanics problems. Computers and Structures, 6: 281-290.

Gaul, L., Kögl, M., and Wagner, M. 2003. Boundary element methods for engineers and scientists. An introductory course with advanced topics. Springer-Verlag, Berlin.

Johnson, K.L. 1985. Contact mechanics. Cambridge University Press, Cambridge, UK.

Lubliner, J.C. 1990. Plasticity theory. Collier-Macmillan, New York.

Michałowski, R., and Mroz, Z. 1978. Associated and non-associated sliding rules in contact friction problems. Archiwum Mechaniki Stoswanej, 30: 259-276.

Murakami, Y. 1987. Stress intensity factors handbook. Vols. 1 and 2. Pergamon Press, Oxford, UK.

Nguyen, T.S., and Selvadurai, A.P.S. 1998. A model for coupled mechanical and hydraulic behaviour of a rock joint. International Journal of Numerical and Analytical Methods in Geomechanics, 22: $29-48$.

Plesha, M.E. 1987. Constitutive models for rock discontinuities with dilation and surface degradation. International Journal for Numerical and Analytical Methods in Geomechanics, 11: 345-362.

Selvadurai, A.P.S. 1991. Non-linear interfaces and fracture mechanics. Wissenschaft Zeitschrift der Hochschule für Architekture und Bauwesen, Weimar, 37: 21-23.

Selvadurai, A.P.S. 1993. Mechanics of a rock anchor with a pennyshaped basal crack. International Journal of Rock Mechanics and Mining Sciences, 30: 1285-1290.

Selvadurai, A.P.S. 1994. Matrix crack extension at a frictionally constrained fiber. Journal of Engineering Materials Technology, Transactions of the ASME, 116: 398-402.

Selvadurai, A.P.S. 1995. Micro-mechanics of a segmented embedded fibre. In Proceedings of the IUTAM Symposium on Anisotropy, Inhomogeneity and Non-linearity in Solid Mechanics, Nottingham, UK, 30 August - 3 September 1994. Edited by D.F. Parker and A.H. England. Kluwer Academic Publishers, Dordrecht, The Netherlands. pp. 391-396. 
Selvadurai, A.P.S. 2000. Fracture evolution during indentation of a brittle elastic solid. Mechanics of Cohesive Frictional Materials, 5: 325-339.

Selvadurai, A.P.S. 2005. Plane cracks with frictionally constrained surfaces. Computers and Structures, 83: 727-739.

Selvadurai, A.P.S., and Au, M.C. 1985. Response of inclusions with interface separation, friction and slip. In Boundary Elements VII: Proceedings of the 7th International Conference, Lake Como, Italy, September. Edited by C.A. Brebbia and G. Maier. Computational Mechanics Publication, Southampton, UK. pp. 14.109-14.127.

Selvadurai, A.P.S., and Au, M.C. 1988. Cracks with frictional surfaces: a boundary element approach. In Proceedings of the 9th Boundary Element Conference. Edited by C.A. Brebbia. Springer-Verlag, Berlin. pp. 211-230.

Selvadurai, A.P.S., and Au, M.C. 1989. Crack behaviour associated with contact problems with non-linear interface constraints. In Boundary Element Techniques: Applications in Engineering, BETECH '89: Proceedings of the 4th International Conference on Boundary Element Technology, Windsor, Ontario, 3-17 June 1989. Edited by C.A. Brebbia and N.G. Zamani. Computational Mechanics Publications, Southampton, UK. pp. 3-17.
Selvadurai, A.P.S., and Boulon, M.J. (Editors). 1995. Mechanics of geomaterial interfaces. Elsevier Scientific Publishing Co., Amsterdam. Studies in Applied Mechanics, No. 17.

Selvadurai, A.P.S., and ten Busschen, A. 1995. Mechanics of the segmentation of an embedded fiber. Part II. Computational modelling and comparison. Journal of Applied Mechanics, Transactions of the ASME, 62: 98-107.

Selvadurai, A.P.S., and Voyiadjis, G.Z. (Editors). 1986. Mechanics of material interfaces. Elsevier Scientific Publishing Co., Amsterdam. Studies in Applied Mechanics, No. 11.

Selvadurai, A.P.S., and Yu, Q., 2005. Mechanics of a discontinuity in a geomaterial. Computers and Geotechnics, 32: 92-106.

Sih, G.C. 1991. Mechanics of fracture initiation and propagation. Kluwer Academic Publishers, Dordrecht, The Netherlands.

Willner, K. 2003. Kontinuums- und kontaktmechanik: synthetische und analytische darstellung. Springer-Verlag, Berlin.

Wriggers, P., and Wagner, W. (Editors). 2000. Computational mechanics: state of the art. Springer-Verlag, Berlin.

Zienkiewicz, O.C., and Taylor, R.L. 2000. The finite element method. Vol. 2. Solid mechanics. Butterworth-Heinemann, Oxford, UK. 